\title{
Cancellation of soft and collinear divergences in noncommutative QED
}

\author{
B. Mirza*, M. Zarei ${ }^{\dagger}$ \\ Department of Physics, Isfahan University of Technology (IUT) \\ Isfahan, Iran,
}

\begin{abstract}
In this paper, we investigate the behavior of non-commutative IR divergences and will also discuss their cancellation in the physical cross sections. The commutative IR (soft) divergences existing in the non-planar diagrams will be examined in order to prove an all order cancellation of these divergences using the Weinberg's method. In noncommutative QED, collinear divergences due to triple photon splitting vertex, were encountered, which are shown to be canceled out by the non-commutative version of KLN theorem. This guarantees that there is no mixing between the Collinear, soft and non-commutative IR divergences.
\end{abstract}

*email: b.mirza@cc.iut.ac.ir

†email: zarei@ph.iut.ac.ir 


\section{Introduction}

Non-commutative field theories have received a great deal of attention [1]. The non-commutativity has also been studied phenomenologically in both high and low energy experiments [2]. These theories, however, suffer from certain shortcomings. Field theories with space-time non-commutativity, for example, do not have a unitary $S$-matrix [3]. The extra branch cuts, in theses theories, are developed in the loop diagrams which are responsible for the failure of the cutting rules and lack of unitary $S$-matrix. It seems that we can save unitarity at the expense of enlarging the Hilbert space of asymptotic states, similar to making the $S$-matrix of open string theory unitary by introducing the closed-string states [4]. The UV/IR mixing observed by Minwalla et al [5] appears to be the main qualitative difference between conventional and non-commutative perturbation field theories. In general, for any non-commutative field theory, the loop diagrams can be classified in the so-called planar and non-planar graphs. Perturbative calculations have shown that the planar diagrams contain exactly the same ultraviolet divergencies as their commutative counterparts do. It has been found that there is an intriguing mix of ultraviolet and infrared scales in the non-planar diagrams. This is what is known as UV/IR mixing, which is a general feature of the non-commutative field theories $[1,5]$. For example, in the noncommutative $\Phi^{4}$ theory it has been found that the effective action has a singularity at $\widetilde{p}=0(\widetilde{p}=p \theta)$ that can be interpreted as an IR divergence at fixed $\theta$ or as a non-analytic behavior in the $\theta \rightarrow 0$ at fixed $p$ [5-7]. The UV/IR mixing in the case of gauge theories also shows some specific features [8-12]. It has been shown that in the non-commutative QED, a new IR divergence (non-commutative IR divergence) appears [ 6]. These noncommutative IR divergences are different from IR (soft) divergences which are related to the existence of massless photons in theory. It has long been known that the soft divergences occurring in QED can be canceled out in transition rates or cross sections computed for detectors with finite energy 
resolution: the soft divergences which occur in a scattering process due to the emission of an undetected soft real photons with total energy $\leq E_{l}$ exactly cancel out the soft divergences due to virtual photon corrections order by order in perturbation theory. This cancellation was first shown by Bloch and Nordsieck 13] in QED and is referred to as Bloch-Nordsieck theorem. In ordinary (commutative) Yang-Mills theories just as in QED, there exist IR (soft) divergences due to massless gluons. In these theories, in addition to the soft divergences, the collinear divergences occur [14]. This kind of divergence is due to the presence of triple gluon vertex. In external lines, gluon can decay into two collinear gluons that leads to collinear divergences. In spite of this, it is believed that the soft collinear divergencies in YangMills theories are canceled out order by order in perturbation theory if the physical cross section is calculated $[15,16]$. This has been guaranteed by the theorems of Kinoshita [17] and of Lee and Nauenberg [18] known as KLN theorem which states that the transition rates are free of the collinear and soft divergencies if we sum over initial and final states. This theorem is a fundamental quantum mechanical theorem on the basis of unitarity of $S$ matrix. A great many studies have investigated the cancellation of collinear and soft divergences at one-loop and two-loop orders [19,20]. In this paper the divergences occurring in non-commutative QED will be explored whose structure is the same as that in Yang-Mills theories. For our purposes, the one-loop non-planar vertex correction will be investigated in order to identify the soft and non-commutative IR divergences as well as the collinear divergence or mass singularity. It will be shown that the soft divergences can be cancelled out by calculating the physical or measured cross section. This method will be applied to the logarithmic non-commutative IR divergences appearing in vertex correction. Another aspect of the present study is to show that this kind of divergence is also canceled out in the physical cross section if the energy resolution of detector is in the order of non-commutative parameter $\theta$. Next, we will turn to an all order soft divergence cancellation using Weinberg's method. This method will be applicable if the triple 
photon vertex is ignored. Otherwise, similar to Yang-Mills theories, the collinear divergences will appear up to the leading order in non-commutative QED. Therefore, it is necessary to deal with the KLN theorem in the noncommutative space. This theorem is proved in the case where unitarity is conserved $\left(\theta_{i 0}=0\right)$. Armed with this proof, the soft and collinear divergences will be shown to cancel out to all orders in the non-commutative QED. Our motivation for considering KLN theorem in a non-commutative space was the following point: "In proceeding to higher loop orders, one faces the danger that the new IR divergences will mix with other divergences in an uncontrollable way, (in page 24 of Douglas and Nekerasov paper,[1])".

This article is organized along the following lines. Section 2 presents the cancellation of soft and and non-commutative IR divergences in vertex correction by computing the physical cross section. Section 3 will provide the proof for the all order cancellation of soft divergences. Finally, in section 4 a non-commutative version of KLN theorem will be introduced and the cancellation of collinear divergences to all orders will be investigated.

\section{Soft and Non-commutative IR Cancellation in the Cross Section}

In this section, we will consider a fermion-fermion scattering in noncommutative QED up to one loop order. The point here is to investigate the cancellation of soft and non-commutative IR divergencies appearing in the non-planar diagrams in the physical cross section. Therefore, non-planar oneloop corrections will be examined and the contribution of the cross section of the elastic scattering will be calculated with the emission of undetected soft photon with an energy $\leq E_{l}$, the energy resolution of the detector. Here an initial electron state $|e\rangle$ is degenerate with the state $|e \gamma\rangle$ (an electron with an emitted photon) when the photon is soft. The diagramatic expression of 
the terms up to order $\alpha^{2}$ in the total cross section of the fermion-fermion scattering is shown in Fig.(1). 

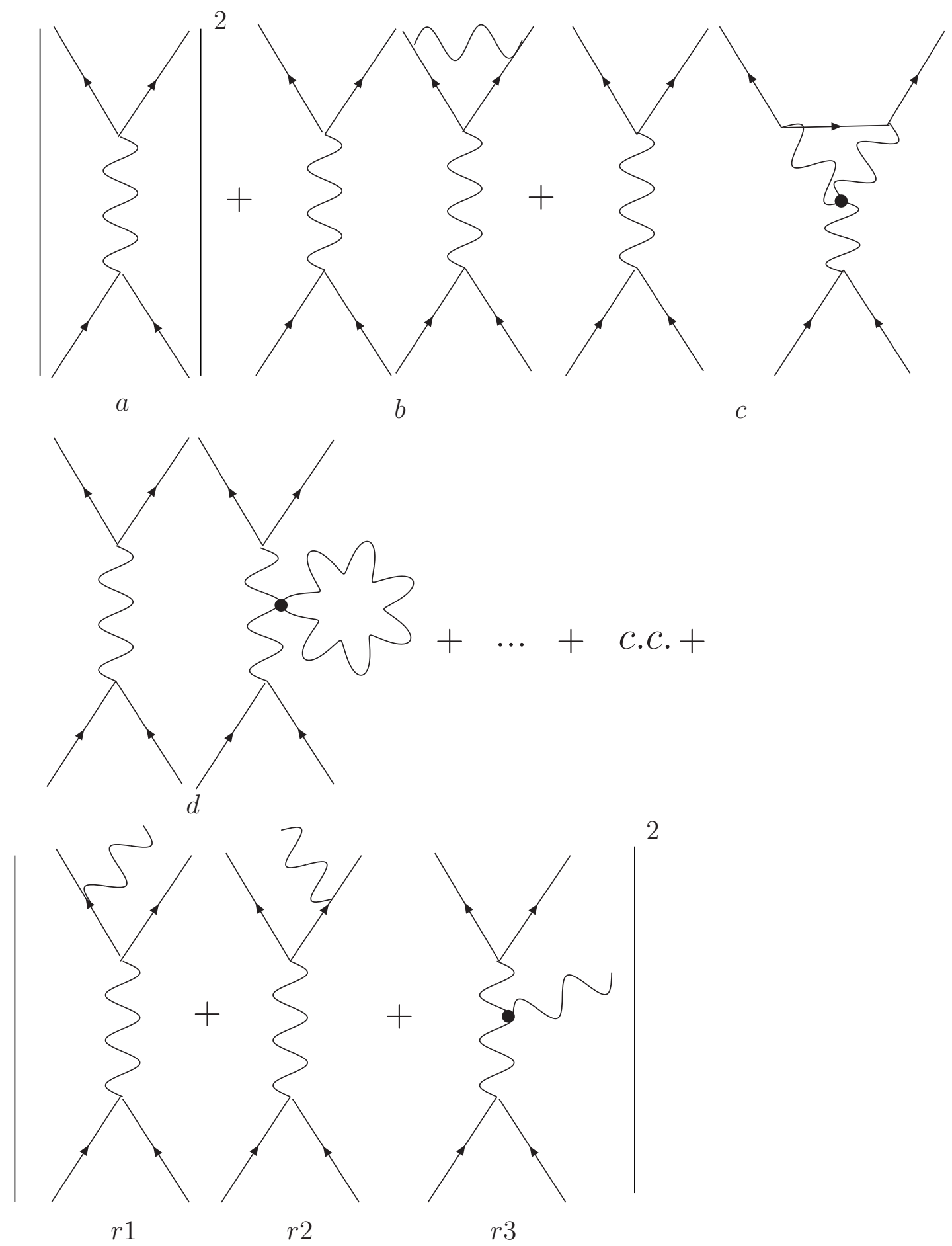
Fig. 1: The diagramatic expression of the terms up to order $\alpha^{2}$ in the total cross section of the fermion-fermion scattering.

Denoting the physical cross section to order $\alpha^{2}$ due to non-planar diagrams, we have

$$
\sigma_{p h}=\sigma_{a}+\sigma_{b}+\sigma_{c}+\sigma_{d}+\cdots+\sigma_{r}
$$

where $\sigma_{a}, \sigma_{b}, \ldots$ are the cross sections associated with diagrammatic expression represented in Fig. $(1 \mathrm{a}, 1 \mathrm{~b}, \ldots)$ and the $\sigma_{r}$ denote the real-photon emission cross section. It is known that non-planar diagrams are UV finite, hence no need for the field renormalization constant. Below is the calculation of amplitudes and the total cross section.

\subsection{Soft, Non-Commutative IR and Collinear Divergencies in the Vertex Correction}

The differential cross section in Fig. $1 \mathrm{~b}$ and its complex conjugate is proportional to quantity $F$ as defined by,

$$
F=\frac{e^{4}}{q^{4}} \operatorname{Tr}\left[\left(\not p_{1}+m\right) \gamma^{\mu}\left(\not p_{2}+m\right) \gamma^{\nu}\right] \operatorname{Tr}\left[\left(\not p_{1}+m\right)\left(\Lambda_{\mu}+\Lambda_{\mu}^{*}\right)\left(\not p_{2}+m\right) \gamma_{\nu}\right]
$$

Where $\Lambda_{\mu}+\Lambda_{\mu}^{*}$ is the one-loop non-planar vertex part and its complex conjugate. Using the Feynman rules for non-commutative QED given in [6] and [21], we will have,

$$
\begin{aligned}
\Lambda_{\mu}+\Lambda_{\mu}^{*} & = \\
& =-e^{2} \int \frac{d^{4} k}{(2 \pi)^{4}} 2 \cos (k \times q) \frac{\gamma_{\sigma}}{k^{2}-\mu^{2}+i \epsilon} \frac{\not \not)^{\prime}-\not k+m}{\left(p^{\prime}-k\right)^{2}-m^{2}+i \epsilon} \gamma_{\mu} \\
& \times \frac{\not p-\not k+m}{(p-k)^{2}-m^{2}+i \epsilon} \gamma^{\sigma}
\end{aligned}
$$

where $k \times q=k_{\mu} \theta^{\mu \nu} q_{\nu}$ and $m$ is the fermion mass and $\mu$ is the fictitious mass for the photon to suppress the soft divergence. Here, the non-commutative 
parameter $\theta^{\mu \nu}$ is assumed to be just magnetic i.e. $\theta^{i 0}=0$. Applying Feynman parameterizatin, we will have,

$$
\Lambda_{\mu}+\Lambda_{\mu}^{*}=-e^{2} \int \frac{d^{4} k}{(2 \pi)^{4}} 2 \cos (k \times q) \frac{\text { numerator }}{D^{3}}
$$

where the denominator $D$ is,

$$
\begin{aligned}
D & =\left(k^{2}-\mu^{2}+i \varepsilon\right) x+\left(\left(p^{\prime}-k\right)^{2}-m^{2}+i \varepsilon\right) y+\left((p-k)^{2}-m^{2}+i \epsilon\right) z \\
& =k^{2}-2 k \cdot(p x+p y)-z \mu^{2}-m^{2}(x+y)+x p^{\prime 2}+y p^{2}+i \varepsilon
\end{aligned}
$$

Now shift $k$ to complete the square,

$$
l \equiv k-x p^{\prime}-y p
$$

After a bit of algebra, drawing on mass-shell condition and $q=p^{\prime}-p$, we will obtain,

$$
D=l^{2}-\Delta+i \epsilon
$$

where

$$
\Delta \equiv x y q^{2}-m^{2}(1-z)^{2}-\mu^{2} z
$$

Based on the on-mass-shell condition and the anti-commutation relation, the numerator in (4) can be written as,

$$
\begin{aligned}
\text { Numerator } & =\bar{u}(p) 4\left[\gamma_{\mu}\left(2 l^{2}\right)-\not l_{\mu}\right]+p_{\mu}\left(4 m x-4 m x y-4 m y^{2}\right) \\
& +p_{\mu}^{\prime}\left(4 m y-4 m x y-4 m x^{2}\right)+\gamma_{\mu}\left[2 m^{2}(x+y)^{2}+m^{2}\right. \\
& \left.-8 m^{2}(x+y)+2 q^{2}(x+y-x y-1)\right] u\left(p^{\prime}\right)
\end{aligned}
$$

At this stage, we will focus on performing momentum integration and handling the terms that will contain the non-commutative IR divergence [6] i.e. the first term. Using the formula given in [22], we can calculate the momentum integration. Below is the final result including the divergent term,

$$
\begin{aligned}
\left(\Lambda_{\mu}+\Lambda_{\mu}^{*}\right)_{N C-I R} & =-e^{2} \int d x d y d z \delta(x+y+z-1) \frac{4 \pi^{2}}{2(2 \pi)^{4}}\left\{\left(-2 \gamma_{\mu}\right) K_{0}(|\widetilde{q}| \sqrt{\Delta})\right. \\
& + \text { finite terms }\}
\end{aligned}
$$


where $|\widetilde{q}|=\sqrt{q_{\mu} \theta^{\mu \nu} \theta_{\nu \lambda} q^{\lambda}}$ and the subscript NC-IR mean the noncommutative IR divergent part. In the $|\widetilde{q}| \rightarrow 0$ limit, we will have a logarithmic divergence,

$$
\begin{aligned}
\left(\Lambda_{\mu}+\Lambda_{\mu}^{*}\right)_{N C I R} & =\frac{\alpha}{\pi} \gamma_{\mu} \int d x d y d z \delta(x+y+z-1) \ln (|\widetilde{q}| \Delta)+ \\
& \text { finite terms }\}
\end{aligned}
$$

Now let us consider the ordinary (commutative) IR divergence in (3). In the $\mu \rightarrow 0$ limit for the last terms in (9) we have,

$$
\begin{aligned}
& -e^{2} \int d x d y d z \delta(x+y+z-1) \frac{4 \pi^{2}}{(2 \pi)^{4}} \frac{1}{\Delta} \gamma_{\mu} \\
& \times\left[2 q^{2}(x+y-x y-1)+m^{2}\left(-8(x+y)+2(x+y)^{2}+1\right)\right]
\end{aligned}
$$

Within this limit, the soft divergences occur in the corner of the Feynman parameter space where $z \approx 1$ (and, therefore, $x \approx y \approx 0$ ). In this region, we can set $z=1$ in the $\mu^{2}$ term in the denominator and for the sake of simplicity, $z=1$ and $x=y=0$ in the numerators. Taking the $x$ integration by defining the new variables, the expression (12) will be simplified to,

$$
\left(\Lambda_{\mu}+\Lambda_{\mu}^{*}\right)_{s o f t}=-\frac{\alpha}{2 \pi}\left(-i e^{2}\right) \log \left(\frac{-q^{2}}{m^{2}}\right) \log \left(\frac{-q^{2}}{\mu^{2}}\right)
$$

There is no effect of non-commutative IR divergences in this region (the soft photon region) just as there are no soft divergences in the noncommutative IR region. The cross section for process shown in Fig.(1a,b) for non-planar diagrams and its complex conjugate is given as,

$$
\left(\frac{d \sigma}{d \Omega}\right)_{\text {total }}=\left(\frac{d \sigma}{d \Omega}\right)_{N C-I R}+\left(\frac{d \sigma}{d \Omega}\right)_{s o f t}
$$

which is equal to,

$$
\left(\frac{d \sigma}{d \Omega}\right)_{B}\left[-\frac{\alpha}{\pi} \int d z d y d z \delta(x+y+z-1) \ln (|\widetilde{q}| \sqrt{\Delta})-\frac{\alpha}{2 \pi} \log \left(\frac{-q^{2}}{m^{2}}\right) \log \left(\frac{-q^{2}}{\mu^{2}}\right)\right]
$$


where $\left(\frac{d \sigma}{d \Omega}\right)_{B}$ denote the Born cross section. Here, certain constant terms have been ignored. In the next step, another divergence namely mass singularity (collinear divergence) will be considered which occurs when mass of the external particles is equal to zero. The non-commutative IR divergence seems to be similar to mass singularity. Taking the $m \rightarrow 0$ limit of (3) we get,

$$
\left(\Lambda_{\mu}+\Lambda_{\mu}^{*}\right)_{\text {collinear }}=-e^{2} \int \frac{d^{4} x}{(2 \pi)^{4}} \frac{1}{k^{2}} \frac{2 \cos (k \times q) N_{\mu}}{\left(k^{2}-2 k \cdot p^{\prime}\right)\left(k^{2}-2 k \cdot p\right)}
$$

where $N_{\mu}$ is as defined in (9). With due attention to the fact that,

$$
\frac{1}{k^{2}}=\frac{1}{\omega}\left(\frac{1}{k_{0}-\omega+i \varepsilon}-\frac{1}{k_{0}+\omega-i \varepsilon}\right)
$$

with $\omega=|\mathbf{k}|$ and performing the $k_{0}$ integration on complex $k_{0}$-plane, we have

$$
\begin{aligned}
\left(\Lambda_{\mu}+\Lambda_{\mu}^{*}\right)_{\text {collinear }}=4 \pi e^{2} & \int_{0}^{\pi} d(\cos \theta) \int \frac{d \omega}{\omega} \cos (\omega \cdot \widetilde{q}) \\
& \times \frac{N_{\mu}^{\prime}}{\left(p_{0}^{\prime}-\left|\mathbf{p}^{\prime}\right| \cos \alpha\right)\left(p_{0}-|\mathbf{p}| \cos \alpha\right)}
\end{aligned}
$$

where $\theta$ is the angle between $\mathbf{p}^{\prime}$ and $\mathbf{k}$ in the center of mass frame. In the $N_{\mu}^{\prime}, k_{0}$ has been replaced with $\omega$. In $m \rightarrow 0$ limit, in which we set the mass of external fermion to zero, we have $p_{0}^{\prime}=\mathbf{p}^{\prime}$ and $p_{0}=\mathbf{p}$. So,

$$
\left(\Lambda_{\mu}+\Lambda_{\mu}^{*}\right)_{\text {collinear }}=4 \pi e^{2} \int \frac{d \omega}{\omega} \cos (\omega \cdot \widetilde{q}) \int_{0}^{\pi} d(\cos \theta) \frac{N_{\mu}^{\prime}}{1-\cos ^{2}(\theta)}
$$

Therefore, under the condition where photon momentum $\mathbf{k}$ is parallel to the external momentum $\mathbf{p}^{\prime}(\mathbf{p})$, we face a divergence in the angular integral or collinear divergence. This kind of divergence is cancelled out in the physical cross section $[17,18]$.

\subsection{Divergence from Real-Photon-Emission}

Now we turn to the calculation of the non-commutative photon emission differential cross section $d \sigma_{r}$. The non-planar diagramatics expression are 
shown in Fig.(1r). In any real experiment, a photon detector can detect photon only down to some minimum energy resolution $E_{l}$. So in a real experiment, a scattered electron can not be distinguished from an electron accompanied by a soft photon with energy down to energy resolution $E_{l}$. We consider the diagrams for the emitted photon from external electron (figs.(1.r1,r2)). The calculation of $d \sigma_{r}$ follows,

$$
\begin{aligned}
d \sigma_{r} & =d \sigma_{B} \int \frac{d^{3} k}{(2 \pi)^{3}} \frac{1}{2 k_{0}} \sum_{\lambda=1,2} e^{2}\left|\int-\frac{p^{\prime} \cdot \epsilon^{(\lambda)}}{p^{\prime} \cdot k} e^{\frac{-i}{2} k \times p^{\prime}}+\frac{p \cdot \epsilon^{(\lambda)}}{p \cdot k} e^{\frac{-i}{2} k \times p}\right|^{2} \\
& =d \sigma_{B} \int \frac{d^{3} k}{(2 \pi)^{3}} \frac{e^{2}}{2 k_{0}} \\
& \times\left[-\frac{m^{2}}{\left(p^{\prime} \cdot k\right)^{2}}-\frac{m^{2}}{(p \cdot k)^{2}}+\frac{2 p \cdot p^{\prime}}{\left(p^{\prime} \cdot k\right)(p \cdot k)} \cos \left(\frac{k \times q}{2}\right)\right]
\end{aligned}
$$

In the extreme relativistic limit, we consider only the third term. As already mentioned, the integration is in the soft region: $0 \leq k_{0} \leq E_{l}$. In order to make the integration well defined, we introduce a very small fictitious mass $\mu$ for the photon which plays the role of a lower cutoff for the integral. So the expression (20) is simplified to,

$$
d \sigma_{r}=d \sigma_{B} \frac{\alpha}{\pi} \int_{\mu}^{E_{l}} \frac{d k_{0}}{k_{0}} I \cos \left(\frac{k \times q}{2}\right)
$$

where

$$
I=\int \frac{d \Omega}{4 \pi} \frac{|\mathbf{k}|^{2}\left(2 m^{2}-q^{2}\right)}{\left(k \cdot p^{\prime}\right)(k \cdot p)}
$$

Note that the quantity $I$ is independent of $k_{0}$; so, by performing the solid angle integration, in the high energy limit $q^{2} \rightarrow \infty$, we obtain,

$$
I=2 \log \left(\frac{-q^{2}}{m^{2}}\right)
$$

Therefore, the cross section $\left(\frac{d \sigma}{d \Omega}\right)_{R}\left(p \rightarrow p^{\prime}+\gamma\left(k<E_{l}\right)\right)$, will read as,

$$
\left(\frac{d \sigma}{d \Omega}\right)_{r}=\left(\frac{d \sigma}{d \Omega}\right)_{B} \frac{\alpha}{2 \pi}\left(\log \frac{E_{l}^{2}}{\mu^{2}} \log \frac{-q^{2}}{m^{2}}\right)
$$


The non-commutative parameter disappears when the integration is performed in the soft region. The cancellation of soft divergence in the vertex correction Fig(1.b) is also to be examined. As discussed earlier, on the scale of ordinary (commutative) QED, the cross section in a scattering event measured by the detector with a specific energy resolution, is given by,

$$
\begin{aligned}
& \left(\frac{d \sigma}{d \Omega}\right)_{\text {measured }}=\left(\frac{d \sigma}{d \Omega}\right)\left(p \rightarrow p^{\prime}\right)+\left(\frac{d \sigma}{d \Omega}\right)\left(p \rightarrow p^{\prime}+\gamma\left(k<E_{l}\right)\right) \\
& =\left(\frac{d \sigma}{d \Omega}\right)_{r}+\left(\frac{d \sigma}{d \Omega}\right)_{s o f t} \\
& =\left(\frac{d \sigma}{d \Omega}\right)_{B}\left[-\frac{\alpha}{2 \pi} \log \left(\frac{-q^{2}}{m^{2}}\right) \log \left(\frac{-q^{2}}{\mu^{2}}\right)+\frac{\alpha}{2 \pi} \log \left(\frac{-q^{2}}{m^{2}}\right) \log \left(\frac{-E_{l}^{2}}{\mu^{2}}\right)\right] \\
& =\left(\frac{d \sigma}{d \Omega}\right)_{B}\left[\frac{\alpha}{2 \pi} \log \left(\frac{-q^{2}}{E_{l}^{2}}\right) \log \left(\frac{-q^{2}}{m^{2}}\right)\right]
\end{aligned}
$$

We see that the soft divergence has been canceled out in this process, which is in agreement with the result from [23]. This is a reliable method that works very well on this scale.

In what follows, this method will be applied to the non-commutative region. In a non-commutative theory, for example non-commutative QED, we investigate the theory on non-commutative scales. So it will be reasonable to set the energy resolution of detector $E_{l}$ in the order of non-commutative parameter $\theta$. We define the "non-commutative soft photon region" as $0 \leq k_{0}=\sqrt{|\mathbf{k}|^{2}+\lambda^{2}} \leq E_{l} \sim \omega^{2}|\widetilde{q}|$, where $\omega$ is a constant with dimension $(\text { energy })^{2}$. In this non-commutative region, a single-electron state can not be distinguished from an electron state accompanied by the soft photon. So for the cross section of the photon emitted from the external electron we have,

$$
d \sigma_{N C-r}=d \sigma_{B} \frac{\alpha}{\pi} \int_{\mu}^{\omega^{2}|\widetilde{q}|} \frac{d k_{0}}{k_{0}} I \cos \left(\frac{k \times k}{2}\right)
$$

The remaining integration is performed in the $\widetilde{q} \rightarrow 0$ and nonrelativistic limit and the final result of the cross section is obtained as follows,

$$
\left(\frac{d \sigma}{d \Omega}\right)_{N C-r}=\left(\frac{d \sigma}{d \Omega}\right)_{B} \frac{\alpha}{\pi} \ln \left(\frac{|\widetilde{q}| \omega^{2}}{\mu}\right)
$$


and therefore,

$$
\left(\frac{d \sigma}{d \Omega}\right)_{\text {measured }} \sim \text { finite. }
$$

in which the measured cross section is free of both soft and non-commutative IR divergences. So because of the existence of non-commutative soft photon, no non-commutative IR divergences of the vertex correction are observed. The quadratic and logarithmic non-commutative IR divergences that comes from new IR non-Abelian vertex and vacuum polarization corrections (Figs. $(1 \mathrm{c}, 1 \mathrm{~d}, \ldots)$ ), may be cancelled out using other methods. For example, one may use the infrared rearrangement method [24] to cancel these non-commutative divergences. This method is used in scalar field theories, where the IR divergences will appear when masses and external momenta are all equal to zero. The non-commutative IR divergences are similar to this kind of divergence.

\section{The Cancellation of the Infrared Divergence to All Orders}

The structure of non-commutative QED is the same as Yang-Mills theories. We expect that at least in non-commutative space $\left(\theta_{i 0}=0\right)$ where the unitarity is confirmed, the soft and collinear divergences are cancelled out to all orders as do in the Yang-Mills theories. In ordinary QED, one approach is the cancellation of soft divergence in on-shell renormalization scheme in which the soft divergence in $Z_{1}$, the vertex rescaling factor cancels against another from $Z_{2}$, the field-strength renormalization constant as a result of the renormalization constant Ward identity $\left(Z_{1}=Z_{2}\right)$ [26]. The result is general and can be extended to all orders. This method is not appropriate in non-commutative QED since in this theory $Z_{1} \neq Z_{2}$ [27. However, we will present an all-order proof of the cancellation using Weinberg's lucid method [28. In this part, the non-planar vertex correction and the terms with the 
largest logarithmic enhancement at each order of perturbation theory will be discussed. It should be noted that the soft divergences from the other non-Abelian diagrams are finite. Let us first consider the outcoming electron line with $n$ soft photon attaching to it as shown in Fig. 2

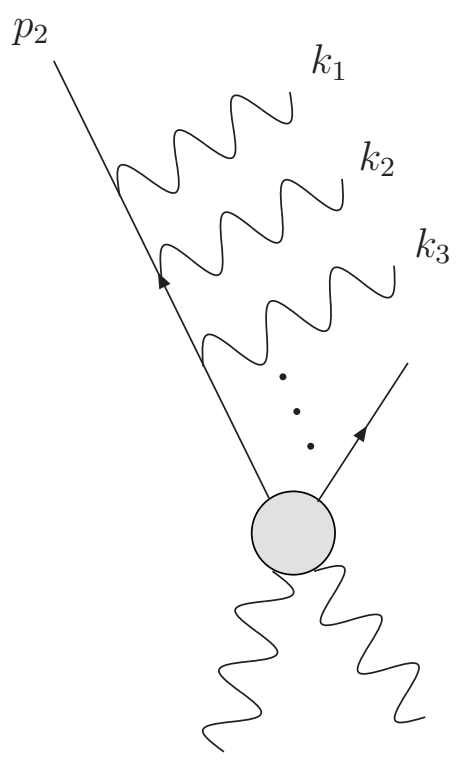

Fig. 2: The $\mathrm{n}$ soft photons attached to an outcoming electron.

The amplitude of this diagram is,

$$
\begin{aligned}
\sum_{\text {perm }} & \bar{u}\left(p_{2}\right)\left(-i e \epsilon_{1}\right) e^{\frac{i}{2} k_{1} \times p_{2}} \frac{i\left(\not p_{2}+\not k_{2}+m\right)}{2 p_{2} \cdot k_{1}} \\
& \times\left(-i e \epsilon_{2}\right) e^{\frac{i}{2}\left(k_{2} \times p_{2}+k_{2} \times k_{1}\right)} \frac{i\left(\not p_{2}+\not k_{1}+\not k_{2}+m\right)}{2 p_{2} \cdot\left(k_{1}+k_{2}\right)} \cdots \\
& \times\left(-i e \epsilon_{n}\right) e^{\frac{i}{2}\left(k_{n} \times p_{2}+k_{n} \times\left(k_{1}+\ldots+k_{n-1}\right)\right)} \frac{i\left(\not p_{2}+\not p_{1}+\ldots+\not k_{n}+m\right)}{2 p \cdot\left(k_{1}+\cdots+k_{n}\right)}\left(i T_{0}\right) \\
& \simeq(e)^{n} \sum_{p e r m} e^{\frac{i}{2}\left[\left(k_{1}+\ldots+k_{n}\right) \times p_{2}+k_{2} \times k_{1}+k_{3} \times\left(k_{1}+k_{2}\right)+\ldots+k_{n} \times\left(k_{1}+\ldots+k_{n-1}\right)\right]} \\
& \times \frac{p_{2} \cdot \epsilon_{1}}{p_{2} \cdot k_{1}} \frac{p_{2} \cdot \epsilon_{2}}{p_{2} \cdot\left(k_{1}+k_{2}\right)} \cdots \frac{p_{2} \cdot \epsilon_{n}}{p_{2} \cdot\left(k_{1}+\ldots+k_{n}\right)} \bar{u}\left(p_{2}\right)\left(i T_{0}\right)
\end{aligned}
$$


where $\epsilon_{i}=\epsilon\left(k_{i}\right)$ is the polarization with $i=1,2, \ldots, n$ and by "perm" we mean all the possible permutations of index $(1,2, \ldots, n)$. Applying the Eikonal approximation, we assume that all the $k_{i} s$ are soft and so drop the $O\left(k^{2}\right)$ terms in the denominators and the $k_{i}$ in the numerator. We must hold the exponential in an all-order treatment; however, the terms like $k_{i} \times k_{j}$ can be ignored (compared to terms $p_{2} \times k_{i}$ in the exponential). The sum of permutation can be performed by means of the following formula,

$$
\sum_{p e r m} \frac{1}{p \cdot k_{1}} \frac{1}{p \cdot\left(k_{1}+k_{2}\right)} \cdots \frac{1}{p \cdot\left(k_{1}+\ldots+k_{n}\right)}=\frac{1}{p \cdot k_{1}} \frac{1}{p \cdot k_{2}} \cdots \frac{1}{p \cdot k_{n}}
$$

The proof of this formula follows from mathematical induction on $n$. Applying (30) to (29), we find the coefficient of $\left(i T_{0}\right)$ as

$$
\bar{u}\left(p_{2}\right) e^{n} \frac{p_{2} \cdot \epsilon_{1}}{p_{2} \cdot k_{1}} \frac{p_{2} \cdot \epsilon_{2}}{p_{2} \cdot k_{2}} \cdots \frac{p_{2} \cdot \epsilon_{n}}{p_{2} \cdot k_{n}} e^{\frac{i}{2} p_{2} \times\left(\sum_{i=1}^{n} k_{i}\right)}
$$

A similar set of manipulations simplifies the sum over soft photon insertion on the incoming electron line (Fig. 3),

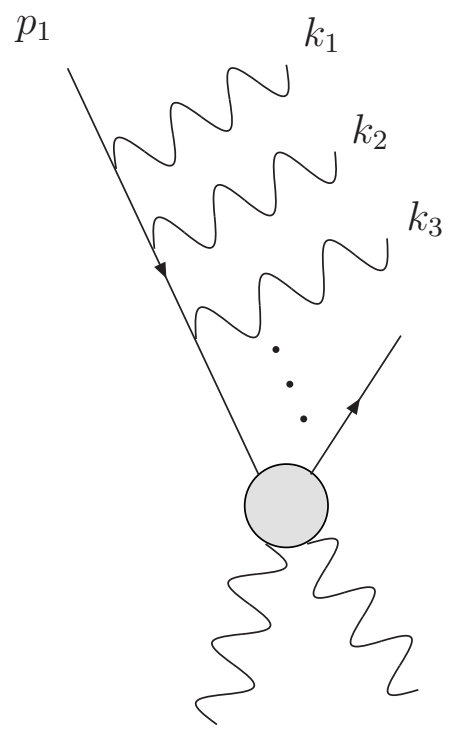


Fig. 3: The $\mathrm{n}$ soft photons attached to an incoming electron.

In this case, we get an extra minus sign in the factor for each photon, since in the denominator we have $\left(p_{1}-\sum k\right)^{2}-m^{2} \approx-2 p \cdot\left(\sum k\right)$. Also in the exponential, we have an extra minus sign. It is now straightforward to obtain the replacement rule for $n$ soft photons attached to $N$ electron lines with all possible partitions $\left(n_{1}, n_{2}, \ldots, n_{N}\right)$ of soft photons with the constraint $\sum_{i=1}^{N}=n$, as shown by a typical diagram in Fig. 4 ,

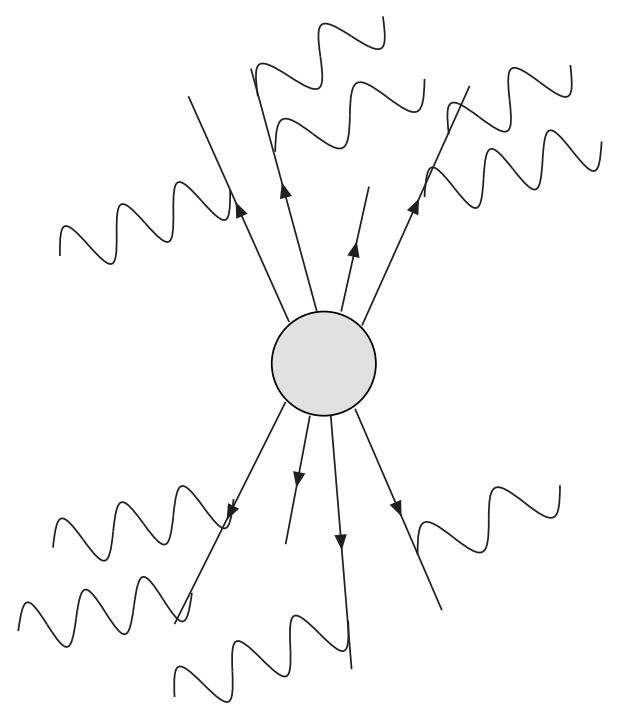

Fig. 4: A typical diagram with $\mathrm{n}$ soft photons attached to $\mathrm{N}$ external electrons.

The extra factor for this attachment is

$$
\sum_{i=1}^{N} \prod_{l=1}^{n_{i}} e \eta_{i} \frac{p_{i} \cdot \epsilon_{l}}{p_{i} \cdot k_{l}} e^{\frac{-i}{2} p_{i} \times \sum_{j=1}^{n_{i}} k_{j}}
$$

where $\eta$ is the signature factor defined by $\eta= \pm 1$ for incoming and outcoming electrons respectively. 
The non-commutative transition amplitude with $N$ external electrons and $n$ soft photons can be written in the following form

$$
T_{n}=T_{0} \prod_{l=1}^{n} e \eta_{i} \frac{p_{i} \cdot \epsilon_{l}}{p_{i} \cdot k_{l}} e^{\frac{-i}{2} p_{i} \times \sum_{j=1}^{n_{i}} k_{j}}
$$

$T_{0}$ is the transition amplitude without photon line in the non-commutative space. The transition rate $\omega_{n}$ for emitting $n$ photons in the non-commutative QED is given by

$$
\omega_{n}=\frac{1}{n !} \int_{R}\left(\prod_{l=1}^{n} \frac{d^{3} k_{l}}{(2 \pi)^{3} 2 k_{l 0}}\right) \sum_{p o l}\left|T_{n}\right|^{2}
$$

where $\sum_{p o l}$ means sum over polarizations and is performed using $-g_{\mu \nu}$ in place of $\sum \varepsilon_{\mu} \varepsilon_{\nu}^{*}$. The soft photon region $R$ is defined as

$$
R=\left\{k_{1}, \ldots, k_{n} ; \sum_{l=1}^{n} k_{l 0} \leq E_{l}\right\}
$$

where $E_{l}$ is the energy resolution of the detector. After summing the polarization, we obtain,

$$
\omega_{n}=\frac{1}{n !}\left|T_{0}\right|^{2} \int_{R} \prod_{l=1}^{n}\left\{\frac{d^{3} k_{l}}{(2 \pi)^{3} 2 k_{l 0}} \sum_{i, j} e^{2} \eta_{i} \eta_{j} \frac{-p_{i} \cdot p_{j}}{\left(p_{i} \cdot k_{l}\right)\left(p_{j} \cdot k_{l}\right)}\right\} e^{\frac{i}{2} \widetilde{Q}_{i j} \cdot \sum_{r=1}^{n} k_{r}}
$$

with $\widetilde{Q}_{i j}=\widetilde{p}_{i}-\widetilde{p}_{j}$. For example, when the number of external electrons is $N=2$, we have $\widetilde{Q}_{i j}=\widetilde{q}$, the momentum transfer. The physical transition rate $\omega$ for $N$-electron process is given by adding all orders,

$$
\begin{aligned}
\omega & =\sum_{n=0}^{\infty} \omega_{n} \\
& =\left|T_{0}\right|^{2} \sum_{n=0}^{\infty} \frac{1}{n !} \sum_{i, j=1}^{N} f \\
& \times \int \frac{d k_{10}}{k_{10}} e^{\frac{i}{2}\left|\widetilde{\mathbf{Q}}_{i j}\right| k_{10} \cos \left(\alpha_{1}\right)} \ldots \frac{d k_{n 0}}{k_{n 0}} e^{\frac{i}{2}\left|\widetilde{\mathbf{Q}}_{i j}\right| k_{n 0} \cos \left(\alpha_{n}\right)} \Theta\left(E_{l}-\sum_{r=1}^{n} k_{r 0}\right)(37
\end{aligned}
$$

where $\alpha_{i}$ is the angle between $\mathbf{k}_{i j}$ and $\widetilde{\mathbf{Q}}_{i j}$. The step function $\Theta$ is defined as,

$$
\Theta\left(\omega-\omega^{\prime}\right)=\frac{-1}{2 \pi i} \int_{-\infty}^{\infty} d x \frac{e^{-i\left(\omega-\omega^{\prime}\right) x}}{x+i \epsilon}=\frac{1}{\pi} \int_{-\infty}^{\infty} d x \frac{\sin \omega x}{x+i \epsilon} e^{i \omega^{\prime} x}
$$


and

$$
f=\int \frac{d \Omega}{2(2 \pi)^{3}} e^{2} \eta_{i} \eta_{j} \frac{-p_{i} \cdot p_{j}}{\left(p_{i} \cdot k\right)\left(p_{j} \cdot k\right)} k_{0}^{2}
$$

Here $d \Omega$ is the $\theta, \phi$ are angles describing $\vec{q}$. By applying Feynman parameterization, it is easy to confirm that the quantity $f$ is independent of $k_{0}$ and is a function of $p_{i} \cdot p_{j}$. It is straightforward to see that the quantity $\widetilde{\mathbf{Q}}_{i j} \cos (\alpha)$ will be cancelled out when we take the integration over $k_{l 0}$,

$$
\begin{aligned}
\int_{\mu}^{E_{l}} \frac{d k_{0}}{k_{0}} e^{i k_{0} x} e^{\frac{i}{2}} \widetilde{\mathbf{Q}}_{i j} \mid k_{0} \cos (\alpha) & =\int_{\mu}^{E_{l}} \frac{d k_{0}}{k_{0}} e^{i k_{0}\left(x+\frac{1}{2}+\left|\widetilde{\mathbf{Q}}_{i j}\right| \cos (\alpha)\right)} \\
& \left.\approx \ln \left(k_{0}\left(x+\frac{1}{2}\left|\widetilde{\mathbf{Q}}_{i j}\right| \cos (\alpha)\right)\right)\right|_{\mu} ^{E_{l}} \\
& =\ln \frac{E_{l}}{\mu}
\end{aligned}
$$

The physical transition rate for emission of any number of soft photons is therefore,

$$
\omega=\left|T_{0}\right|^{2} \frac{1}{\pi} \int_{-\infty}^{\infty} d x \frac{\sin x}{x+i \varepsilon} \exp \left(f \ln \left(\frac{E_{l}}{\mu}\right)\right)=\left|T_{0}\right|^{2}\left(\frac{E_{l}}{\mu}\right)^{f}
$$

Let us now consider the situation in which there is no external photon line. We turn to the virtual photon line participating only as an internal line in $T_{0}$. Denoting by $t_{n}$ the transition amplitude of $N$-electron with insertion of $n$ internal photon lines (Fig. 5), we have,

$$
T_{0}=\sum_{n=0}^{\infty} t_{n}+\text { finite terms }
$$




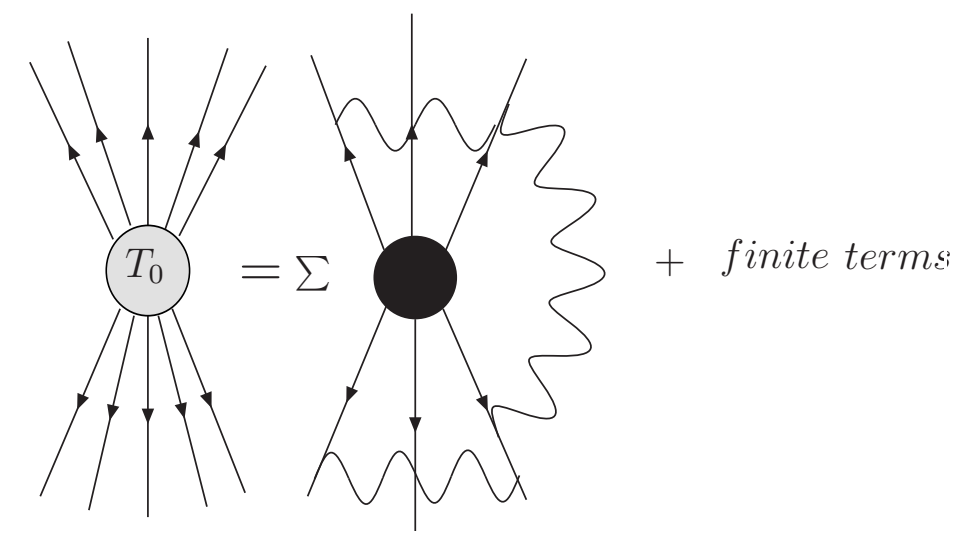

Fig. 5: Diagramatic expression of the fact that the soft divergences emerge only through the internal photon lines.

For each virtual photon attached to two external electron lines (Fig. 5) we obtain the following expression for the amplitude

$$
\begin{aligned}
\frac{e}{2} \int \frac{d^{4} k}{(2 \pi)^{4} i} \bar{u}\left(p_{i}\right) \gamma_{\mu} \frac{1}{m-\not p_{i}+\not k} M_{0} \frac{1}{m-\not p_{j}-q} e^{\frac{i}{2} \widetilde{Q}_{i j} \cdot k} \gamma_{\nu} u\left(p_{j}\right) \frac{g^{\mu \nu}}{k^{2}} \\
\approx \frac{e^{2}}{k^{2}} \int_{V} \frac{d^{k}}{(2 \pi)} \frac{1}{k^{2}} \frac{p_{i} \cdot p_{j}}{\left(p_{i} \cdot k\right)\left(p_{j} \cdot k\right)} M_{0} e^{\frac{i}{2} \widetilde{Q}_{i j} \cdot k}
\end{aligned}
$$

where $V$ denotes the soft photon region $\mu \leq k_{0} \leq \Lambda$ and $t_{0} \equiv \bar{u}\left(p_{i}\right) M_{0} u\left(p_{j}\right)$ is the amplitude corresponding to the core diagram (The dark blob in Fig. 5 , indicates that part of the full diagram having no infrared divergences and is called the core diagram). The factor $\frac{1}{2}$ is required since our procedure counts each Feynman diagram twice. The explicit expression for $t_{1}$ and thus $t_{n}$ reads in general as,

$$
t_{1}=t_{0} \int \frac{d^{4} k}{(2 \pi)^{4} i} \frac{e^{2}}{k^{2}+i \varepsilon} \frac{1}{2} \sum_{i, j}^{N} \frac{-\eta_{i} \eta_{j} p_{i} \cdot p_{j}}{\left(p_{i} \cdot k\right)\left(p_{j} \cdot k\right)} e^{\frac{i}{2} \widetilde{\mathbf{Q}}_{i j} \cdot k}
$$

and

$$
t_{n}=t_{0} \frac{1}{n !}\left[\int \frac{d^{4} k}{(2 \pi)^{4} i} \frac{e^{2}}{k^{2}+i \varepsilon} \frac{1}{2} \sum_{i, j}^{N} \frac{-\eta_{i} \eta_{j} p_{i} \cdot p_{j}}{\left(p_{i} \cdot k\right)\left(p_{j} \cdot k\right)} e^{\frac{i}{2} \widetilde{\mathbf{Q}}_{i j} \cdot k}\right]^{n}
$$


where the factor $\frac{1}{n !}$ comes as a result of symmetry considerations in attaching the $n$ internal photon to external electron lines. From (42), equation (45) leads to

$$
T_{0}=t_{0} e^{\frac{g}{2}}
$$

where the factor $g$ is defined as,

$$
g=\int \frac{d^{4} k}{(2 \pi)^{4} i} \frac{2 e^{2}}{k^{2}+i \varepsilon} \sum_{i, j}^{N} \frac{-\eta_{i} \eta_{j} p_{i} \cdot p_{j}}{\left(p_{i} \cdot k\right)\left(p_{j} \cdot k\right)} e^{\frac{i}{2} \widetilde{\mathbf{Q}}_{i j} \cdot k}
$$

Squaring $T_{0}$, we have,

$$
\left|T_{0}\right|^{2}=\left|t_{0}\right|^{2} e^{R e g}
$$

Using the fact that,

$$
\frac{1}{k^{2}+i \varepsilon}=\frac{P}{k^{2}}-i \pi \delta\left(k^{2}\right)
$$

with $P$ indicating the principal value, we have,

$$
\begin{aligned}
\operatorname{Reg} & =\int \frac{d^{4} k}{(2 \pi)} 2 e^{2} \pi \delta\left(k^{2}\right) \sum_{i, j}^{N} \frac{\eta_{i} \eta_{j} p_{i} \cdot p_{j}}{\left(p_{i} \cdot k\right)\left(p_{j} \cdot k\right)} \sin \left(\frac{1}{2} \widetilde{\mathbf{Q}}_{i j} \cdot \mathbf{k}\right) \\
& =-\int_{\mu}^{\Lambda} \frac{d k_{0}}{k_{0}} \sin \left(\frac{1}{2} \widetilde{\mathbf{Q}}_{i j} \cdot k_{0}\right) f \approx-f \ln \left(\frac{\Lambda}{\mu}\right)
\end{aligned}
$$

where $f$ is as defined by Eq.(39), so

$$
\left|T_{0}\right|^{2}=\left|t_{0}\right|^{2}\left(\frac{\mu}{\mu}\right)^{f}
$$

Now with inserting $\left|T_{0}\right|^{2}$ into the equation (41), we confirm the finiteness of the physical transition rate as $\mu \rightarrow 0$,

$$
\omega=\left|t_{0}\right|^{2}\left(\frac{\mu}{\Lambda}\right)^{f}\left(\frac{E_{l}}{\mu}\right)^{f}=\left|t_{0}\right|^{2}\left(\frac{E_{l}}{\Lambda}\right)^{f}
$$

This cancellation can also be checked order by order. Note that in the above argument we have confined ourselves to the soft photon region in which the non-commutative IR divergence disappears. Note also that the 
diagrams involving triple photon emission have not been dealt with here. Such processes are completely novel and lead to collinear divergences whose cancellation requires such general theorems as KLN. In the next section we turn to this theorem in non-commutative space.

\section{The Kinoshita-Lee-Nauenberg Theorem in Non-Commutative Space}

We showed that in the non-commutative theories with massless gauge fields as commutative ones, there are both IR and mass divergences. In some Feynman diagrams with triple photon splitting, there exists another kind of collinear divergences. Some methods have been introduced to cancel these divergences in Yang-Mills theory, for example in QCD, a constructive approach leading to a soft-finite asymptotic dynamics which produces a set of asymptotic states with a $S$-matrix operator with soft finite elements [29]. These states are essential generalizations of the coherent states. This is a perturbative procedure and has been developed in analogy with that used in the Abelian case. Moreover, there is a fundamental quantum mechanical theorem greatly advanced by the works of Kinoshita [17] and Lee and Nauenberg [18] (KLN theorem [25]) that provide the standard theoretical response to the problem of collinear and soft divergences. For clarity, we assume the problem contains a certain parameter $\mu$ and the degeneracy occurs in the total Hamiltonian

only when $\mu \rightarrow 0$ [25]. For example in non-commutative QED $\mu$ can be mass of the photon.

As they have shown, the occurrence of such divergences are consequence of high degree of degeneracy in the system. The cancellation of these divergences can be established without any explicit use of Feynman graghs, nor even the explicit form of the Hamiltonian [17,18,25].

This theorem and also the coherent state approach are based on unitarity. In this paper, we will try to prove the non-commutative version of KLN 
theorem on the condition that unitarity is conserved i.e. $\theta_{i 0}=0$. This theorem reads as follows :

In a non-commutative field theory with only space non-commutativity with massless fields, transition rates are free of the soft and collinear divergence if the summation over the initial and final degenerate states is carried out.

The degenerate states means, for example, the states with an electron and soft photon belonging to the same energy eigenstate as that of a single electron in the limit of vanishing photon energy. The proof of non-commutative version of KLN theorem is straightforward. We begin by investigating the structure of $S$-matrix in the interaction picture on noncommutative space. In the interaction picture, the Schrödinger equation for the state $|\alpha, t\rangle$ in the natural unit system is,

$$
\begin{gathered}
i \frac{\partial}{\partial t}|\alpha, t\rangle=g \widehat{H}_{I}^{(\theta)}|\alpha, t\rangle \\
\widehat{H}_{I}^{(\theta)}=e^{i H_{0} t} H_{I}^{(\theta)} e^{-i H_{0} t}
\end{gathered}
$$

where $H_{0}$ is the free Hamiltonian and $H_{I}{ }^{\theta}$ is the interaction term in which the common product between fields has been replaced by $\star$-product. For example, in the field theory context $g H_{I}^{(\theta)}$ can be of the forms $e \int d^{3} x \gamma^{\mu} \bar{\psi} \star$ $A_{\mu} \star \psi$. Defining a unitary operator

$$
U\left(t, t_{0}\right)=T\left\{\exp \left(-i \int_{t_{0}}^{t} d \lambda \widehat{H}_{I}^{(\theta)}(\lambda)\right)\right\}
$$

where $T$ denotes the time-order product, the time evolution of the state $|\alpha, t\rangle$ can be described as

$$
|\alpha, t\rangle=U\left(t, t_{0}\right)\left|\alpha, t_{0}\right\rangle
$$

Also the $U\left(t, t_{0}\right)$ satisfies the same differential equation,

$$
i \frac{\partial}{\partial t} U\left(t, t_{0}\right)=g \widehat{H}_{I}^{(\theta)} U\left(t, t_{0}\right)
$$

Now with the initial condition $U\left(t_{0}, t_{0}\right)=1$, equation (57) can be solved to obtain the $S$-matrix which is defined as [30],

$$
S=U(-\infty, \infty)
$$




$$
\begin{aligned}
& =\sum_{n=0}^{\infty} \frac{(-i)^{n}}{n !} \int_{-\infty}^{\infty} d t_{1} \int_{-\infty}^{\infty} d t_{2} \ldots \int_{-\infty}^{\infty} d t_{n} T\left\{\widehat{H}_{I}^{(\theta)}\left(t_{1}\right) \widehat{H}_{I}^{(\theta)}\left(t_{2}\right) \ldots \widehat{H}_{I}^{(\theta)}\left(t_{n}\right)\right\} \\
& =T\left\{\exp \left(-i \int d t \widehat{H}_{I}^{(\theta)}(t)\right)\right\}
\end{aligned}
$$

Providing that $\theta_{i 0}=0$, the $S$-matrix is unitary: $S^{\dagger} S=S S^{\dagger}=1$. So also, we have

$$
S=U(-\infty, 0) U(0, \infty)=U^{\dagger}(0,-\infty) U(0, \infty)
$$

So for the transition rate $|\langle f|S| i\rangle|^{2}$, we get

$$
\left|\left\langle f\left|U^{\dagger}(0,-\infty) U(0, \infty)\right| i\right\rangle\right|^{2}
$$

In order to see the origin of the IR divergence from the initial and final degenerate states explicitly, we separate the transition rate into two parts: one including only the final state $|f\rangle$ and the other including the initial state $|i\rangle$,

$$
\left|\left\langle f\left|U^{\dagger}(0,-\infty) U(0,+\infty)\right| i\right\rangle\right|^{2}=\sum_{m} \sum_{n}\left(R_{m n}^{+}\right)^{*} R_{m n}^{-}
$$

where

$$
\begin{gathered}
R_{m n}^{+}=\langle m|U(0,+\infty)| f\rangle^{*}\langle n|U(0,+\infty)| f\rangle \\
R_{m n}^{-}=\langle m|U(0,-\infty)| i\rangle^{*}\langle n|U(0,-\infty)| i\rangle
\end{gathered}
$$

For problems in field theory, one may imagine (61) as cutting rules. Now let us examine the problem by expanding $U$ up to order $g$ and performing the time integration, from which we will have

$$
\begin{gathered}
R_{m n}^{+}=\delta_{m f} \delta_{n f}-\frac{g\left\langle m\left|H_{I}^{(\theta)}\right| f\right\rangle^{*}}{E_{m}-E_{f}-i \varepsilon} \delta_{n f}-\frac{g\left\langle n\left|H_{I}^{(\theta)}\right| f\right\rangle}{E_{n}-E_{f}+i \varepsilon} \delta_{m f} \\
R_{m n}^{-}=\delta_{m i} \delta_{n i}-\frac{g\left\langle m\left|H_{I}^{(\theta)}\right| i\right\rangle^{*}}{E_{m}-E_{i}+i \varepsilon} \delta_{n f}-\frac{g\left\langle n\left|H_{I}^{(\theta)}\right| f\right\rangle}{E_{n}-E_{i}-i \varepsilon} \delta_{m i}
\end{gathered}
$$

where $E_{r}$ is the eigenvalue of $H_{0}$ for the state acting on state $|r\rangle$. In an example of scattering, the states $|m\rangle$ and $|f\rangle$ in $R^{+}$may be an electron state $|e\rangle$ and the state including an electron and an emitting photon $|e \gamma\rangle$, 
respectively. If the emitting photon is soft or collinear, the states $|e\rangle$ and $|e \gamma\rangle$ will have the same energy eigenvalues and are degenerate. So in (64) and (65) the denominators will be divergent and we face an infrared divergence. Suppose $D(E)$ is a subspace of the Hilbert space with all degenerate states with energy $E(\mu \rightarrow 0)$.

We may consider the case of soft divergence in non-commutative QED. So the parameter $\mu$ can be the photon mass and $D(E)$ can be consisted of any photon whose energy is $<E_{l}$. Then in the quantity

$$
\sum_{m \in D(E)} \sum_{n \in D(E)}|\langle m|S| n\rangle|^{2}
$$

the IR divergences are absent. To prove this from (61), it is sufficient to show

that $\sum_{r \in D(E)} R_{m n}^{ \pm}$is free from the IR divergence. The proof is easy and to order $g$ and all orders of $\theta$ the non-commutative parameter is given from

$$
\begin{aligned}
\sum R_{m n}^{ \pm} & =0 \text { if } m, n \notin D(E) \\
& =-\frac{g\left\langle m\left|H_{I}^{(\theta)}\right| n\right\rangle^{*}}{E_{m}-E \mp i \varepsilon} \text { if } m \notin D(E) \text { and } n \in D(E) \\
& =-\frac{g\left\langle n\left|H_{I}^{(\theta)}\right| m\right\rangle}{E_{n}-E \pm i \varepsilon} \text { if } m \in D(E) \text { and } n \notin D(E) \\
& =0 \text { if } m, n \in D(E)
\end{aligned}
$$

Hence $R_{m n}^{ \pm}$(and consequently Eq. (66)) are finite up to order g. The generalization of this proof to all orders of $g$ is not difficult. It should be noted that in the above we have essentially used the unitarity of the operator $U$; i.e., the condition $\theta_{i 0}=0$. The non-commutative KLN theorem guarantees the cancellation of both collinear and soft divergences at each order of perturbation theory.

\section{Conclusion}

In this paper, the possibility of the non-commutative IR divergence cancellation at one-loop order in physical cross sections was studied. We 
defined a non-commutative soft photon which the detector could not see in the non-commutative scales. It has been demonstrated that in a scattering process computed up to one-loop order, if we consider the non-commutative soft photon emission, the non-commutative logarithmic IR divergence in the vertex correction will be cancelled in the cross section. However, there are additional non-Abelian type diagrams in which their non-commutative logarithmic and quadratic divergences can not be canceled out using the cross section method. This non-cancellation is attributed to an important difference between soft and non-commutative IR divergences. The soft divergences are associated with the classical limit but non-commutative IR divergences are completely a quantum mechanical effect. We have further shown the soft divergences that only appear in the non-planar vertex correction to be cancelled out in the physical cross section to all orders. To prove this cancellation, the Weinberg's method was used. In an all order treatment, we also have examined the diagrams involving the triple photon splitting that led to collinear divergences similar to Yang-Mills theories. Some general theorems such as KLN guarantee that the transition rates are free from this kind of divergence if we sum over initial and final degenerate states. In this paper, we studied the KLN theorem in the non-commutative space $\left(\theta_{i 0}=0\right)$. According to this theorem, the non-commutative QED will be free of collinear divergence.

\section{References}

[1] M.R. Douglas and N.A. Nekrasov, Rev. Mod. Phys. 73(2001)977, hep-th/0106048; R.J. Szabo, Phys. Rept. 378 (2003) 207-299, hep-th/0109162 N. Seiberg, E. Witten, JHEP9909(1999)032, hep-th/9908142.

[2] M. Chaichian, A. Demichev, P. Presnajder, M.M. Sheikh-Jabbari, A. Tureanu, Phys. Lett. B527(2002)149, hep-th/0012175. M. Chaichian, 
M.M. Sheikh-Jabbari, A. Tureanu, Phys.Rev.Lett.86(2001)2716, hep-th/0010175 B. Mirza, M. Zarei, Eur. Phys. J. C 32(2004)583, hep-th/0311222 I. Hinchliffe, N. Kersting, Y.L. Ma, Int. J. Mod. Phys. A 19 (2004)179, hep-ph/0205040

[3] J. Gomis and T. Mehen, Nucl. Phys. B 591 (2000) 265-276 hep-th/0005129.

[4] L. Alzarez-Gaume, J.L.F Barbon and R. Zwicky, JHEP 0105 (2001)057, hep-th/0103069.

[5] S. Minwalla, M. Raamsdonk and N. Seiberg, JHEP 0002 (2000) 020. hep-th/9912072.

[6] A. Mika and M.M. Sheikh-jabbari, JHEP 0101 (2001) 025, hep-th/0008057.

[7] I.Ya. Aref'eva, D.M. Below and A.S. Koshele, Phys. Lett. B476 (2000) 431, hep-th/9912075.

[8] M. Hayakawa, Perturbation analysis of IR aspects of noncommutative QED on $R^{4}$, Phys. Lett. B478 (2000)394, hep-th/9912094.

[9] A. Matusis, L. Susskind and N. Toumbas, The IR/UV connection in noncommutative gauge theories, JHEP 0012 (2000) 002, hep-th/0002075.

[10] L. Alvarez-Gaume and M. A. Vazquez-Mozo, hep-th/0311244.

[11] C.P. Martin and F. Ruiz, Paramagnetic dominance, the sign of beta function and UV/IR mixing in noncommutative U(1), Nucl. Phys.B 597 (2001) 197, hep-th/0007131.

[12] J. Ambjorn, Y.M. Makeenko, J. Nishimura, R.J. Szabo, Lattice gauge fields and discrete doncommutative Yang-Mills theory, JHEP 0005 (2000) 023, hep-th/0004147 
[13] F. Bloch and A. Nordsieck, Phys. Rev. 52, 54(1937).

[14] For a resent review see: M. Lavelle and P. McMullan, Collinearity, convergence and cancelling infrared divergence, hep-ph/0511314.

[15] F.G. Krausz, Nucl. Phys. B 126 (1977) 340; F.G. Krausz, Phys. Lett. 66B (1977) 251.

[16] T. Appelquist, J. Carazzone, H. Kluberg-Stern and M. Roth, Phys. Rev. Lett.36, 768 (1976); L. Tyburski, Phys. Rev. Lett. 37, 319 (1976); Y.P.Yao, Phys. Rev. Lett. 36, 653 (1976).

[17] T. Kinoshita, J. Math. Phys. 3 (1962) 650.

[18] T.D. Lee and M. Nauenberg, Phys. Rev. 133 (1964) 1549.

[19] T. Appelquist and J. Carazzone, Nucl. Phys. B120 (1977) 77; C.P.K Altes and E.de Rafael, Nucl. Phys. B 125 (1977) 275; J. Frenkel, R. Meulbemans, I. Mohammad and C.J. Taylor, Nucl. Phys. B 121 (1977) 58; A. Sugamoto, Phys. Rev D16(1977) 1065

[20] T. Muta, Foundations of Quantum Chromodynamice, world scientific, 1998.

[21] I.F.Riad and M.M. Sheikh-jabbari, Noncommutative QED and anomalous magnetic moment, JHEP. 08 (2000) 45, hep-th/0008132.

[22] F.T. Brandt, A. Das and J. Frankel, Phys. Rev. D65 (2002) 085017, hep-th/0112127.

[23] K.B. Eom, S.S. Kong, B.H. Lee and C. Park, Radiative correction in noncommutative QED, hep-th/0205093

[24] A.A. Vladimirov, Theo. Math. Phys. 36, 732 (1978).

[25] T.D. Lee, Particle Physics and Introduction to field theory, Harwood Academic Publishers (1981), (see pages 725-743). 
[26] G. Sterman, An Introduction to Quantum Field Theory, Cambridge University Press, 1994.

[27] C.P. Martin and D. Sanchez-Ruiz, Phys. Rev. Lett. 83, 476 (1999), hep-th/9903077.

[28] S. Weinberg, Phys. Rev. 140 B(1965) 516.

[29] C.A. Nelson, Nucl. Phys. B 181 (1981) 141; Nucl. Phys. B186 (1981) 187.

[30] A.P. Balachandran, T.R. Govindarajan, C. Molina, P. TeotonioSobrinho, JHEP 0410 (2004) 072. 